\title{
PSICOLOGIA
}

\section{SAÚDE E QUALIDADE DE VIDA: INFLUÊNCIA DO STRESS NO AMBIENTE DE TRABALHO}

\author{
DOI: http://dx.doi.org/10.31072/rcf.v8i2.547 \\ HEALTH AND LIFE QUALITY: THE INFLUENCE OF STRESS IN THE WORK \\ ENVIRONMENT
}

Jayne Gomes'; Adriana Souza da Silva²; Gésica Borges Bergamini³.

RESUMO: O estresse pode ser compreendido como estado que se coloca o organismo diante de mudanças gerando instabilidade, o stress inicia a partir de reações emocionais que desencadeiam disfunções fisiológicas e psicológicas. E a Qualidade de Vida no Trabalho e definida como a humanização na organização proporcionando condições para o individuo se desenvolver pessoalmente no melhor nível de bem-estar. Objetivos: Compreender o conceito de qualidade de vida no trabalho definidos por diferentes autores; analisar os efeitos do stress no ambiente de trabalho e sua influência para desencadear psicopatologias do trabalho. Método: Pesquisa bibliográfica foi direcionada através de artigos digitais e livros virtuais anexados ao Google acadêmico, em plataformas de bases de dados Scientific Electronic Library Online (SCIELO), Periódico eletrônico de psicologia (PEPSIC). Resultados e discussão: Através da pesquisa e o estudo bibliográfico do tema foi possível determinar a influencia notável do stress no ambiente de trabalho no comprometimento da saúde mental; e as noções básicas para alcançar uma qualidade de vida no local de trabalho e os métodos que as organizações podem adotar para chegar a nível suficiente de qualidade de vida obtendo resultados satisfatórios no desenvolvimento organizacional. Os principais métodos apresentados são propor a organização estar desenvolvendo um plano de saúde, com benefícios e dias de laser com os próprios membros do grupo e com a família, colocar metas com objetivos específicos levantando um prêmio às pessoas que mais se desempenham em tal proposta. Ver o colaborador como ser ativo de potenciais e não apenas como um recurso institucional.

Palavras-chave: Qualidade de vida. Stress. Ambiente de trabalho.

\footnotetext{
1 Acadêmica de Psicologia da Faculdade de Educação e Meio Ambiente - FAEMA. E-mail: jaynegomes9@gmail.com. ORCID: https://orcid.org/0000-0001-8753-7991;

2 Acadêmica de Psicologia da Faculdade de Educação e Meio Ambiente - FAEMA. E-mail: adriana.souza.97@outlook.com. ORCID: https://orcid.org/0000-0003-0560-0278;

${ }_{3}^{3}$ Professora Mestra do Departamento de Psicologia da Faculdade de Educação e Meio Ambiente - FAEMA. Orientadora desta pesquisa. E-mail: gpensemagro@gmail.com. ORCID: https://orcid.org/0000-0003-05985366.
} 
ABSTRACT: Stress can be understood as the state that puts the body in the face of changes generating instability, stress starts from emotional reactions that trigger physiological and psychological dysfunctions. And the Quality of Life in Work is defined as the humanization in the organization providing conditions for the individual to develop personally in the best level of well-being. Objectives: To understand the concept of quality of life at work defined by different authors; to analyze the effects of stress on the work environment and its influence in triggering work psychopathologies. Method: Bibliographic research was directed through digital articles and virtual books attached to academic Google, on platforms of Scientific Electronic Library Online (SCIELO), Electronic Psychology Journal (PEPSIC). Results and discussion: Through the research and the bibliographic study of the subject it was possible to determine the notable influence of stress in the work environment in the commitment of mental health; and the basics to achieve a quality of life in the workplace and the methods that organizations can adopt to reach a sufficient level of quality of life obtaining satisfactory results in organizational development. The main methods presented are to propose that the organization is developing a plan of health, with benefits and laser days with the members of the group and with the family, to set goals with specific objectives by raising a premium to the people who perform the most in such proposal. See the collaborator as being potential asset and not just as an institutional resource.

Keywords: Quality of life. Stress. Work environment.

\section{INTRODUÇÃO}

A qualidade de vida no contexto contemporâneo está conceituada a vínculos de satisfação entre a relação de satisfação do trabalhador com as atividades exercidas no ambiente de trabalho, atendendo as necessidades e pretensões humanas. Desse modo, compreender o conceito de qualidade de vida, saber como alcançar a satisfação dos profissionais tanto no nível biológico quanto psicológico e pessoal no conjunto organizacional, torna-se necessário e de primordial importância.

A proposta desta pesquisa foi de entender os processos que formam os objetivos específicos e próprios de cada organização, para obter uma melhor qualidade de vida no ambiente de trabalho, realizando levantamento de índices e condições do stress que podem influenciar na qualidade de vida e desempenho profissional. Como resultado desta pesquisa apresenta-se algumas estratégias para evitar um ambiente estressante e ausente de patologias do trabalho.

Kurogi(1) relata que as interações estabelecidas entre trabalhadores dentro das organizações e no contexto social se transformam constantemente. Acompanhar tais mudanças é de fundamental importância para a permanência de uma organização no mercado. Ou seja, manter um ambiente saudável determina estar em harmonia com e através de seus colaboradores, vinculando ambientes motivacionais que 
promovem a determinação das pessoas para alcançar um objetivo conjunto.

Para Sadir, Bignotto \& Lipp ${ }^{(2)}$ além de identificar e compreender a determinação do trabalho nas condições de vida constitui um eixo importante para verificar como o trabalhador percebe e organiza suas relações com o mundo "fora do trabalho", contemplando 0 acesso aos recursos sociais, de educação, saúde, lazer, entre outros.

Camelo \& Angerami ${ }^{(3)} 0$ stress tem sido considerado como uma preocupação, por estar vinculado a várias consequências que podem acarretar dificuldades em uma melhor qualidade de vida do ser humano. Diante desta problemática a qualidade de vida se tornou um tema em debate para a sociedade e na literatura científica, buscando compreender todos os fatores influenciáveis, como o stress, que abrem caminho para ocorrência de inúmeras patologias que trazem sofrimento psíquico e físico para o individuo.

Com isso o trabalho buscou descrever os aspectos influenciáveis do stress no ambiente de trabalho que desempenham 0 comprometimento da qualidade de vida. $\mathrm{E}$ os efeitos do stress no desencadeamento de psicopatologias e efeitos prejudiciais a saúde corporal e psicológica, pois a organização deve estar envolvida de forma estrutural aos membros para proporcionar o crescimento pessoal e profissional é assim elaborar estratégias que possam ser executadas para chegar a uma qualidade de vida digna e favorável para todos os colaboradores da organização.

\section{REVISÃO DE LITERATURA}

\subsection{Saúde e qualidade de vida no ambiente de trabalho}

Segundo Vasconcelos ${ }^{(4)}$, no que se se refere à qualidade de vida destaca-se o a teoria de Abraham Maslow, na teoria da pirâmide de necessidades. Onde concebe a hierarquia das necessidades, composta por cinco necessidades fundamentais: fisiológica, segurança, amor, estima e auto-realização. Nesta teoria demonstra a ligação entre os objetivos individuais e os organizacionais, que ambos devem andar lado a lado. Compreendendo o ambiente onde se relaciona, com relações interpessoais, as condições de trabalho, salários, status e segurança no trabalho. $O$ ambiente de trabalho deve estar ligado a todos esses fatores motivacionais, que vão contribuir e abranger as realizações, reconhecimento do próprio trabalho, responsabilidade e progresso ou desenvolvimento tanto individual quanto organizacional.

Albuquerque \& França(5) define a qualidade de vida no trabalho como o conjunto das ações de uma empresa que 
envolve a implantação de melhorias e inovações gerenciais e tecnológicas no ambiente de trabalho. A construção da qualidade de vida no trabalho ocorre a partir do momento em que se olha a empresa e as pessoas como um todo, o que chamamos de enfoque biopsicossocial.

Segundo Bergamini(6) o indivíduo propõe no seu trabalho diferentes aspectos dependendo do papel exercido na organização e o contexto no qual se insere o que gera várias facetas e formas de relações de trabalho. $\mathrm{Na}$ sociedade atual as pessoas constroem nas organizações suas identidades e adquirem suas próprias vivências por ser nesse local onde se passa maior parte do tempo da vida humana, compreendendo o conceito de dinâmica organizacional e fundamental tanto para o colaborador quanto para a organização.

Os autores Souza \& Figueiredo(7) refere-se que o conceito de qualidade de vida vem sendo utilizado nos campos da saúde e do trabalho a fim de verificar variáveis presentes nos diversos contextos sociais que possam sofrer intervenção através das políticas de saúde ou de estratégias de gestão empresarial. Estudos sobre o impacto da organização e do trabalho sobre a saúde e qualidade de vida do trabalhador apresentam múltiplas dimensões de análise.

Segundo Sadir, Bignotto \& Lipp ${ }^{(2)}$ :

Qualidade de vida é um estado de
bem-estar físico, mental e social e não
somente a ausência de doenças. As
pessoas que se consideram felizes
atribuem sua felicidade ao sucesso em
quatro áreas (social, afetiva, saúde e
profissional).

Diversas pesquisas como de Sadir, Bigotto \& Lipp ${ }^{(2)}$ demostram que a partir do momento que a organização visualiza o trabalhador como colaborador do alcance de seus objetivos organizacionais, tem-se um aumento na qualidade de vida no trabalho. Buscando focalizar o trabalhador na organização, visando a assegurar-lhe melhores resultados de produção, melhor ambiente de trabalho, mais estabilidade na organização, melhores condições de trabalho e, consequentemente, aumento da produtividade.

\subsection{Influências do stress no ambiente de trabalho}

Segundo Carvalho \& Serafim ${ }^{(8)}$, um dos meios de sobrevivência do indivíduo é o trabalho, pois é através dele que se é possível conseguir meios para sobrevivência, principalmente alimentação e moradia. É nesse ambiente laboral que as pessoas passam a maior parte do seu dia, sendo de grande valia ter suas funções, objetivos pessoais e profissionais 
bem definidos e traçados no contexto organizacional.

De acordo com Kanaane ${ }^{(9)} \mathrm{o}$ trabalho sempre ocupou lugar central nas comunidades, sendo limitado pelas condições socialmente estabelecidas. Por meio do trabalho o indivíduo pode se autorealizar, exercer sua capacidade criativa e estabelecer relacionamentos interpessoais gratificantes. Por outro lado, o trabalho pode também ser fonte de adoecimento quando, por exemplo, contém fatores de risco para a saúde e o trabalhador não dispõe de estratégias para se proteger destes riscos, sejam eles físicos ou psicológicos. Para Murta \& Tróccoli(10), há alguns trabalhos, atuações laborais, que de certa forma, coloca a vida dos operários em risco, através de más condições e de pouca segurança no âmbito de trabalho.

A segurança no âmbito trabalhista é algo que tem apresentado uma relevância muito grande, afinal, a instituição que não preza pela segurança e bem-estar de seus trabalhadores, principalmente as que apresentam fatores de risco, acaba regredindo em seu faturamento. Mas claro, que não podemos esquecer que muitas das vezes a negligencia em relação à segurança no trabalho parte do próprio funcionário que se nega a utilizar os acessórios necessários, na maioria das vezes essa negação é encontrada pelo ambiente estressante (11).

De acordo com Paul E. Spector em seu livro Psicologia organizações uma grande dificuldade da prevenção dos acidentes no ambiente de trabalho é conquistar a cooperação dos funcionários na utilização dos equipamentos de segurança apropriados e no envolvimento em comportamentos seguros. As pessoas muitas vezes consideram os equipamentos de segurança incômodos e desconfortáveis.

Algumas vezes as pessoas inutilizam - mecanismo de liberação de empunhadura de um cortador de grama por considerarem-no incomodo e deixam de usar óculos de proteção porque são desconfortáveis. Convenções adotadas informalmente entre funcionários podem excluir a utilização de determinadas praticas por serem consideradas um desperdício de tempo, por demandarem muito esforço ou até por refletir falta de coragem de enfrentar situações perigosas (11).

Continuando com os estudos de Paul E. Spector, ele aponta a importância do clima de segurança de trabalho nas organizações onde procedimentos, práticas e comportamentos seguros são encorajados e recompensados pelos superiores, reflete tanto na politicas das 
organizações quanto nas praticas relevantes a segurança, como na utilização de equipamentos protetores ${ }^{(11)}$.

Para Spector (11) o estresse no trabalho e fora dele pode ser outro importante fator, como foi dito a cima. $\mathrm{O}$ modo como às pessoas se sentem em relação ao emprego e suas atitudes no que $s$ refere à segurança também afetam acidentes e lesões. Pessoas satisfeitas com o trabalho e pessoas que adotam atitudes positivas no que se refere à segurança são menos propensas a sofrer acidentes no trabalho. Ou seja, pessoas que encontram uma satisfação ao seu ambiente trabalhista, onde conseguem manter uma qualidade de vida adequada e conciliando sua vida pessoal, não tendo um índice de estresse elevado, possui uma capacidade de manter um ambiente de trabalho livre de problemas de saúde tanto física como psicológico, evitando até adversidade como 0 estresse póstraumático encontrado principalmente em serviços como bombeiros, policiais, enfermeiros, etc. que demandam de um ambiente mais hostil e nocivo à própria vida.

Segundo Seligmann-Silva (12) e colaboradores, a globalização financeira e a mundialização da precarização social, juntamente com as inovações tecnológicas e as novas formas de gestão, causaram rápidas transformações no mundo do trabalho. As condições trabalhistas vêm sofrendo diversas modificações, que em muitos casos, acabam sendo prejudiciais para a saúde do trabalhador, devido à carga estressante que o mesmo tem que enfrentar em seu ambiente de trabalho. Os aspectos sociais, econômicos e organizacionais, assim como os processos psicossociais em suas repercussões sobre a subjetividade, são minimizados ou ignorados.

Para Camelo \& Angerami (3), o trabalho, nos dias de hoje, parece ser um importante fator gerador de estresse, pois, tem sido considerado como um dos problemas que mais frequentemente agem sobre o ser humano, e interfere na homeostase, ou seja, do equilíbrio de seu organismo, devido à grande quantidade de tensões que enfrenta diariamente. Quando o episódio estressante é de longa duração, as consequências sobre 0 organismo podem ser mais intensas, levando ao desgaste progressivo e, às vezes, ao esgotamento, 0 que evidentemente compromete a desempenho do trabalhador além do âmbito do trabalho, trazendo consequências intensas para a vida pessoal do indivíduo. O estresse é considerado uns dos geradores principais de psicopatologia encontrada no contexto ocupacional. 
A Organização Internacional do Trabalho (OIT) (3) relata que os riscos psicossociais encontrados no trabalho consistem, por um lado, na interação entre o trabalho, seu ambiente, a satisfação no trabalho e as condições de sua organização; por outro, nas capacidades do trabalhador, suas necessidades, sua cultura e sua situação pessoal fora do trabalho, o que, afinal, através de percepções e experiências, pode influir na saúde e no rendimento do trabalhador. Portanto, para se tiver uma boa qualidade de saúde no ambiente trabalhista, o trabalhador deve se encontrar dentro de organização, onde alcance 0 contentamento, respeito e 0 reconhecimento de que, além de trabalhador, faz parte da instituição como um todo, o sentido de pertencimento a este meio social.

Glowinkowski \& Cooper (13), afirmam que os estressores podem ser fatores intrínsecos ao trabalho, os quais se referem a aspectos como repetição de tarefas, pressões de tempo e sobrecarga. Dentre eles, a sobrecarga de trabalho tem recebido considerável atenção dos pesquisadores. Este estressor pode ser dividido em dois níveis: quantitativo e qualitativo. A sobrecarga quantitativa diz respeito ao número excessivo de tarefas a serem realizadas; isto é, a quantidade de tarefas encontra-se além da disponibilidade do trabalhador. A sobrecarga qualitativa refere-se à dificuldade do trabalho, ou seja, o indivíduo depara-se com demandas que estão além de suas habilidades ou aptidões.

De acordo Bergamini (6) uma organização que não remete a uma qualidade de vida adequada a seus funcionários é considera uma organização nociva, onde leva o adoecimento físico e psicológico de seus operários. As más condições podem ser vistas através da falta de preocupação com a saúde, falta de segurança, cargas horárias exaltantes, falta da disseminação da cultura organizacional, gestores despreparados, falta de respeito aos profissionais, inexistência de uma política de comunicação interna específica e ausência da qualidade de vida no trabalho. Esses são alguns dos fatores encontrados dentro de uma instituição nociva. Não há clima organizacional adequado e positivo se a empresa não investe na melhoria da qualidade de vida do trabalho, é impossível ter uma boa relação no ambiente de trabalho sem estabelecer meios para melhor á qualidade de vida. Para isso, não são obrigatórios grandes investimentos. Um ambiente que se apresenta com pinturas suaves, realização 
de alguns eventos comemorativos, um envio de e-mail para quem está aniversariando, promoção de palestras de interesse geral dos colaboradores são algumas ações simples que agregam valor à vida pessoal e profissional de qualquer indivíduo.

Para Vasconcelos (4) não se pode remediar a execução que as demandas e pressões ambientais têm sobre as empresas de uma forma genérica, nem se pode julgar e/ou prescrever estratégias ambientais gerais para elas sem considerar todos os elementos de seu contexto, sem uma análise detalhada de toda a instituição, ou o setor que apresenta uma "infecção". Ou seja, implica também que se deve ter um olhar cuidadoso com as tipificações de estratégias ambientais empresariais, que classificam as empresas ou os seus comportamentos como proativos, reativos, etc.

\section{MATERIAIS E MÉTODOS,}

O presente trabalho teve como método a pesquisa bibliográfica, usando como descritores: qualidade de vida, stress, ambiente de trabalho. Direcionando para pesquisas sobre a qualidade de vida de colaboradores em organizações e a influência do stress no ambiente de trabalho. Destacando como objetivo de entender tais causas provenientes para elevar o stress como principal forma de ocorrências patológicas em organizações. Através de artigos digitais de plataformas virtuais, usando o Google Acadêmico como principal fonte de dados selecionouse os trabalhos mais pertinentes à temática para síntese e apresentação das informações.

\section{RESULTADOS/DISCUSSÕES}

A qualidade de vida no trabalho é um tema que vem sendo bastante questionado, afinal, como conseguir manter a homeostase com o âmbito do trabalho e sua vida pessoal?

Esse assunto remete a integridade física e psíquica do sujeito, já que passamos a maior parte da nossa vida dentro do ambiente de trabalho. Com isso, quais as possíveis psicopatologias podem surgir a partir de um estresse muito elevado nesse local? Quais os mecanismos encontrados para continuar a se manter dentro de uma organização, que gradativamente, vai ceifando a seriedade física e psicológica do indivíduo, quando o local se torna nocivo?

Segundo Carvalho \& Serafim ${ }^{(8)}$, o trabalho em si, com a evolução da globalização, tecnologia e o capitalismo, ao longo da história, passou a ser a ocupação mais presente na vida do ser humano. O que de princípio era para prover as necessidades básicas de subsistência passou a ser o epicentro da 
vida do homem. Portanto, é nesse ambiente que o indivíduo passa a destinar suas capacidades cognitivas, força, energia e esforço. Por decorrência dessa dominação do trabalho, sobre o sujeito, que visa à preocupação com a qualidade de vida, dentro e fora, do âmbito organizacional, pois, um recinto ocupacional prejudicial pode levar qualquer um a desenvolver patologias físicas e psicológicas que iram influenciar seu desenvolvimento na instituição e sua vida pessoal.

Para Freudenberger \& Richelson (14), as exigências das instituições para com os trabalhadores, por conta do ambiente organizacional e a globalização em si, vêm se tornando cada vez mais presentes. Essa busca por resultados torna-se um desafio, aumentando a cobrança de suas capacidades e ocasionando um constante estresse emocional, por conta da pressão imposta pelas demandas internas da organização. Além dessa continua pressão presente no ambiente trabalhista, imposta por uma organização nociva, existem outros fatores como, a carga horária, ambiente de trabalho inadequado, superiores perversos, dentre outros, que são responsáveis pelo acréscimo do estresse que podem influenciar, além dos sintomas tradicionais, outras psicopatologias, como ansiedade, depressão, síndrome de burnout, ou esgotamento profissional, decorrente ao stress prolongado no trabalho.

Freudenberger \& Richelson descreveram um indivíduo com burnout como estando frustrado ou com fadiga, uma sensação de desgaste, cansaço e falta de energia, desencadeada pelo investimento em determinada causa, modo de vida ou relacionamento que não correspondeu às expectativas. A síndrome de burnout pode ser encontrada em indivíduos que se encontram no seu limite no âmbito de trabalho.

De modo que Abreu (15), a exaustão emocional é caracterizada por um sentimento muito forte de tensão emocional que produz uma sensação de esgotamento, de falta de energia e de recursos emocionais próprios para lidar com as rotinas da prática profissional e representa a dimensão individual da síndrome. A despersonalização é o resultado do desenvolvimento de sentimentos $\mathrm{e}$ atitudes negativas, por vezes indiferentes e cínicas em torno daquelas pessoas que entram em contato direto com o profissional, que são sua demanda e objeto de trabalho. Num primeiro momento, é um fator de proteção, mas pode representar um risco de desumanização, constituindo a dimensão interpessoal de burnout. Por último, a falta 
de realização pessoal no trabalho caracteriza-se como uma tendência que afeta as habilidades interpessoais relacionadas com a prática profissional, o que influi diretamente na forma de atendimento e contato com as pessoas usuárias do trabalho, bem como com a organização. Trata-se de uma síndrome na qual o trabalhador perde o sentido da sua relação com o trabalho, de forma que as coisas não the importam mais e qualquer esforço the parece inútil. Finalmente, a síndrome de burnout tem sido negativamente relacionada com saúde, performance e satisfação no trabalho, qualidade de vida e bem-estar psicológico Rabin.

Segundo Santos (16), o sofrimento do trabalhador não fica restrito ao espaço físico do local de trabalho e suas relações, ao contrário espalham-se a todas as suas relações sociais. $\mathrm{O}$ adoecimento no âmbito do trabalho provoca várias alterações na vida do trabalhador e estas alterações repercutirão psicossocialmente além dos limites do trabalho afetando assim toda a vida do trabalhador e ocasionando angustias rotineiras.

Heloani \& Capitão ${ }^{(17)}$, na maioria das vezes 0 trabalhador não possui a consciência de que seu ambiente de trabalho esteja sendo prejudicial a sua saúde física, ou psicológica. Por decorrência do medo existente do desemprego, muitos ignoram os sintomas predominantes e encontram meios de se alto manipularem. Essa manipulação, ou auto-enganação, é conhecida como mecanismo de defesas segundo Dejours ${ }^{18}$. Atualmente, observa-se uma pressão constante contra a grande massa de trabalhadores existente em quase todo 0 mundo. Uma ameaça com objetivo certeiro faz com que milhares de pessoas sintamse sobressaltadas, pois a única ferramenta de que dispõem sua força de trabalho, pode ser dispensada a qualquer momento.

O trabalhador na busca de reduzir sua angustia adota estratégias de defesas que fortalece a alienação e impossibilidade da criatividade, trazendo prejuízos não somente para si próprio como, se distância afetivamente do seu processo produtivo, se fechando para capacidade reflexiva do contexto no qual faz parte e se acomoda diante de uma situação que não the traz benefício algum. Neste cenário a organização também perde, visto que, diante das constantes demandas de mercado necessitarão de mudanças, inovações e criatividade.

Segundo Santiago (19), os recursos chamados de estratégias defensivas, pode ser tanto individual quanto coletivas. As estratégias individuais de defesa são caracterizadas pelos mecanismos de 
defesa operantes, os quais estão interiorizados e operam mesmo sem a presença do outro. Essas estratégias possuem importante papel para a adaptação ao sofrimento, porém são de natureza individual, não atuando sobre a violência social. Segundo Dejours ${ }^{18}$ já as estratégias coletivas de defesa necessitam de um consenso do grupo e dependem de condições externas ao sujeito. Essas estratégias são construídas por um grupo de trabalhadores para resistir aos efeitos desestabilizadores e para lidar com as contradições advindas do trabalho.

Para Dejours (18), elas contribuem para a coesão do coletivo de trabalho. Mendes ${ }^{20}$ relata que as defesas podem ser pensadas tanto como fatores de alienação por não atuarem na modificação da realidade que faz sofrer $e$, consequentemente, possíveis causadores de adoecimento, como podem também ser pensadas como aquelas que desempenham um papel considerável para a manutenção da saúde, por minimizarem a percepção que 0 trabalhador tem do sofrimento. Quando levantados quesitos de qualidade de vida, que colocam a produtividade e eficiência organizacional, como metas prioritárias para a organização, desencadeiam fatores que produzem pressão contínua no ambiente de trabalho também contribui para a decadência da qualidade de vida do trabalhador além de redução da qualidade do trabalho realizado.

Apesar de algumas organizações apresentarem culturas nocivas ao trabalhador, outras buscam formas que possam ajudar em uma boa qualidade de vida de seus funcionários. É de suma importância que as instituições adquirem estratégias, que se consiga estabelecer um âmbito motivacional satisfatório para todos os envolvidos, fazendo assim, alcançar metas através da harmonia dentro da mesma. Uma instituição que visa à qualidade de vida de seus trabalhadores garante a eficácia e produtividade $e$, ao mesmo tempo, 0 atendimento das necessidades básicas dos trabalhadores. Instituições que alcançam intervenções neste sentido priorizam a qualidade do ambiente em que seus funcionários estão inseridos e seu bem-estar durante a realização de suas tarefas ${ }^{(12)}$.

De acordo com Fernandes (21), a Qualidade de Vida no Trabalho pode ser utilizada para que as organizações renovem suas formas de organização no trabalho, de modo que, ao mesmo tempo em que se eleve o nível de satisfação do pessoal, se eleve também a produtividade das empresas, como resultado de maior participação dos empregados nos processos relacionados ao seu trabalho. 
Segundo Albuquerque e LimongiFrança (5), a Qualidade de Vida no Trabalho é um conjunto de ações de uma empresa que envolvem diagnósticos e implantação de melhorias e inovações gerenciais, tecnológicas e estruturais dentro e fora do ambiente de trabalho, visando a propiciar condições plenas de desenvolvimento humano para e durante a realização do trabalho.

A Psicologia Organizacional e do Trabalho (POT), não apenas como um campo de atuação e formação profissional, mas também como campo de produção de conhecimento e diagnostico, tem apresentado uma eficácia dentro das instituições que buscam aprimorarem ações e encontrar estratégias de produção para a qualidade de vida dos funcionários inseridos.

Com a ajuda de um psicólogo organizacional e do trabalho as organizações iriam adquirir estratégias onde ajudará no desenvolvimento, tanto da empresa, como a da saúde de seus funcionários. Através da observação do ambiente, iria apresentar maneiras de aperfeiçoamento do trabalho, o aprimoramento em suas atividades estruturais com 0 desenvolvimento de técnicas, modos de organização e produção, apresentaria técnicas de motivação para evitar o adoecimento do trabalhador e visaria à qualidade de vida, não só dos trabalhadores, mas da organização com um todo.

\section{CONSIDERAÇÕES FINAIS}

Portanto através da pesquisa bibliográfica foi possível compreender a grande influencia do stress no comprometimento da qualidade de vida e saúde do trabalhador. $E$ os fatores organizacionais, ambientais e comportamentais podem manter a tal qualidade de vida perante o processo organizacional de crescimento de todos os membros nesse conjunto reconhecimento de estar em um trabalho em equipe.

Então se faz necessário que as empresas não se preocupem apenas em alcançar os objetivos de lucro, mas que comecem a priorizar a qualidade no ambiente que é oferecido aos colaboradores. E ações que sejam projetadas para levar em consideração o grupo, e não o individual ou setorial.

Assim promover a sensibilização e estímulo a um estilo de vida saudável. Para empresas que adotam tais praticas é natural à diminuição de acidentes no trabalho, redução nos gastos com serviços médicos, diminuição de faltas de trabalhadores por motivo de doenças, aumento na produção das empresas e na satisfação do empregado. $O$ trabalho proporcionou enriquecer o conhecimento 
cientifico para com o tema, elevando os acadêmicos a necessidade de elaboração de novos estudos para formar ainda mais informações comprovadas por teoria e prática.

\section{REFERÊNCIAS}

1. Kurogi MS. Qualidade de vida no trabalho e suas diversas abordagens. Revista de Ciências Gerenciais 2015; 12(16), 63-76.

2. Sadir MA, Bignotto MM, Lipp NME. Stress e qualidade de vida: influência de algumas variáveis pessoais. Paideia 2010; 20(45), 73-81.

3. Camelo SHH, Angerami ELS. Riscos psicossociais no trabalho que podem levar ao estresse: uma análise da literatura. Ciência, Cuidado e Saúde 2008; 7(2), 234-240.

4. Vasconcelos AF. Qualidade de vida no trabalho: origem, evolução e perspectivas. Caderno de pesquisas em Administração 2001; 8(1), 23-35.

5. Albuquerque LG, França ACL. Estratégias de recursos humanos e gestão da qualidade de vida no trabalho: o stress e a expansão do conceito de qualidade total. Revista de Administra\&ccdeil; ão da Universidade de São Paulo 1998; 33(2).

6. Bergamini GB, Aloise MO. Trabalho e o Seu Papel Social Uma Perspectiva Existencial do Individuo Social. [citado em 7 de novembro de 2017]. Disponível em: http://artigos.netsaber.com.br/resumo_artig o_20060/artigo_sobre_o-trabalho-e-o-seupapel-social.

7. Souza LB, Figueiredo MAC. Qualificação profissional e representações sobre trabalho e qualidade de vida. Paidéia 2004; 14(28), 221-232.

8. Carvalho AV, Serafim OCG. Administração de recursos humanos. Pioneira 1998.
9. Kanaane R. Comportamento humano nas organizações: o homem rumo ao século XXI. In Comportamento humano nas organizações: o homem rumo ao século XXI. Atlas 1995.

10. Murta SG, Tróccoli BT. Avaliação de intervenção em estresse ocupacional. Psicologia: teoria e pesquisa 2004; 20(1), 39-47.

11. Spector PE. Psicologia nas organizações. Cap. 11 Psicologia da saúde ocupacional. 4.ed. São Paulo (SP), Saraiva; 2012. p. 294-324.

12. Seligmann-Silva E, Hespanhol Bernardo M, Maeno M, Kato M. O mundo contemporâneo do trabalho e a saúde mental do trabalhador. Revista Brasileira de Saúde Ocupacional 2010; 35(122), 187191.

13. Glowinkowski SP, Cooper CL. Managers and professionals in business/industrial settings: the research evidence. In J. M. Ivancevich \& D.C. Gangster (Orgs.), Job stress: from theory to suggestion. Nova York:Haworth 1987; 8(2), 177-194.

14. Freudenberger HJ, Richelson G. Estafa: $O$ alto custo dos empreendimentos. Rio de Janeiro: Francisco Alves 1991.

15. Abreu KLD, Stoll I, Ramos LS, Baumgardt RA, Kristensen $\mathrm{CH}$. Estresse ocupacional e Síndrome de Burnout no exercício profissional da psicologia. Psicologia: ciência e profissão 2002; 22(2), 22-29.

16. Santos LS. Psicopatologias relacionadas ao Trabalho. Universidade gama filho. [citado em 7 de novembro de 2017]. Disponível em: 
http://www.avm.edu.br/monopdf/23/LORE NNA\%20SOLIVA\%20DOS\%20SANTOS.p $\mathrm{df}_{\text {. }}$

17. Heloani JR, Capitão CG. Saúde mental e psicologia do trabalho. São Paulo em perspectiva 2003; 17(2), 102-108.

\section{Dejours C. Banalização da Injustiça} Social, a. FGV Editora, 1999.

19. Santiago EB. O ambiente de trabalho, as estratégias de defesas e suas implicações na construção da identidade do trabalhador. Encontro: Revista de Psicologia 2015; 16(25), 9-29.

20. Mendes AM. Da psicodinâmica à psicopatologia do trabalho. In A $M$. Mendes (Ed.), Psicodinâmica do trabalho: Teoria, métodos e pesquisa. São Paulo, SP: Casa do Psicólogo 2007.

21. Fernandes EC. Qualidade de vida no trabalho. Salvador: Casa da qualidade 1996; 21-30.

22. Areias MEQ, Guimarães LAM. Gênero e estresse em trabalhadores de uma universidade pública do estado de São Paulo. Psicologia em estudo 2004.
23. Souza AA, Fernandes ACP. Qualidade de vida no trabalho. J. Health Sci. Inst 2013; 31(1).

24. Freire MG. Qualidade de vida no trabalho 2013.

25. Santos JA. Qualidade de vida no trabalho 2014.

26. Suzuke HH, Morés G. Qualidade de vida no trabalho. Revista Eletrônica Interdisciplinar 2016; 2(16).

27. Campos MO, Rodrigues Neto JF. Qualidade de vida: um instrumento para promoção de saúde. Revista Baiana de saúde pública 2014; 32(2), 232.

28. Oliveira EA. Delimitando o conceito de stress. Ensaios e Ciência 2006; 1(1), 11 18.

29. Wanderley DN. Qualidade de vida no trabalho: psicopatologias do trabalho e seu impacto nas organizações. 2016.

30. Silva PC; Merlo ÁRC. Prazer e sofrimento de psicólogos no trabalho em empresas privadas. Psicologia: Ciência e profissão, v. 27, n. 1, p. 132-147, 2007.

\section{Como citar (Vancouver)}

Gomes J, Silva AS, Bergamini GB. Saúde e qualidade de vida: influência do stress no ambiente de trabalho. Rev Cient Fac Educ e Meio Ambiente [Internet]. 2017;8(2):207-220. DOI: http://dx.doi.org/10.31072/rcf.v8i2.547 